\section{High Electron Mobilities Achieved with an Organic Field-Effect Transistor Material}

In the December 4 issue of Applied Physics Letters, researchers J.H. Schön, Ch. Kloc, and B. Batlogg of Bell Laboratories report room-temperature electron mobilities as high as $5.5 \mathrm{~cm}^{2} / \mathrm{Vs}$ for an organic field-effect transistor (OFET) based on single crystalline perylene. Perylene, also called peri-dinaphthalene, has a formula of $\mathrm{C}_{20} \mathrm{H}_{12}$; the structure shows four 6-carbon rings surrounding, and sharing a side with, a central cyclohexane ring. This promising material shows both $n$ - and $p$ channel activities, with the electron mobility clearly exceeding the hole mobility across the 50-300 K temperature range studied. In previous investigations, OFET materials consistently showed hole mobilities that were higher than electron mobilities.

Single crystals of perylene approximately $1-\mathrm{cm}^{2}$ in area and a few micrometers thick were grown from the vapor phase in a stream of flowing hydrogen. Gold source and drain contacts were thermally deposited on the perylene layer, and a dielectric layer of $\mathrm{Al}_{2} \mathrm{O}_{3}$ was sputtered over the surface. A gold gate electrode was evaporated on top of the structure. Structures fabricated included channel lengths from 25 to $50 \mu \mathrm{m}$ with channel widths from 500 to $1,500 \mu \mathrm{m}$.

Standard FET equations yielded electron mobilities of $5.5 \mathrm{~cm}^{2} / \mathrm{Vs}$ and hole mobilities of $0.4 \mathrm{~cm}^{2} / \mathrm{Vs}$, in good accordance with time-of-flight values reported for perylene single crystals. The investigators believe the $5.5 \mathrm{~cm}^{2} / \mathrm{Vs}$ value to be the highest room-temperature mobility reported to date for an OFET in either polarity mode.

The temperature-dependence of the mobility for electrons followed a power law across the whole 50-300 K temperature range, with mobility increasing with decreasing temperature. The hole mobility demonstrated similar behavior down to $60 \mathrm{~K}$, at which point the mobility leveled off. The researchers attributed this phenomenon to defects, disordering, or trapping processes.

\section{TIM PALUCKA}

\section{Individual Layer Thickness of Sol- Gel-Derived BST Thin Films Directs Film Properties}

A team of researchers from the Shanghai Institute of Technical Physics (affiliated with the Chinese Academy of Sciences) and Shandong University has shown that the structure, ferroelectricity, and dielectric properties of sol-gelderived $\mathrm{Ba}_{0.8} \mathrm{Sr}_{0.2} \mathrm{TiO}_{3}$ (BST) thin films strongly depend on the individual layer thickness. In the October issue of the Journal of the American Ceramics Society, the researchers report that the films prepared with an individual layer thickness of $60 \mathrm{~nm}$ showed small equiaxed grains with a cubic structure, no ferroelectricity, and temperature-independent dielectric behavior. In contrast, films prepared with a layer thickness of $8 \mathrm{~nm}$ consisted of columnar grains with a tetragonal crystal structure and showed good ferroelectricity as well as two peaks in the dielectric constant-temperature curve. According to the researchers, the individual layer thickness for layer-by-layer homoepitaxy growth of BST films should be $<20 \mathrm{~nm}$ in the sol-gel processing.

While BST is a promising material for dynamic random access memory (DRAM) and uncooled infrared detector applications due to its high dielectric constant and composition-dependent Curie tempera- ture, sol-gel-derived BST thin films have not produced pronounced ferroelectric hysteresis loops. According to Jun-Hao $\mathrm{Chu}$, director of the National Laboratory of Infrared Physics at the Shanghai Institute of Technical Physics, this failure renders such films unsuitable for infrared detector applications. His team therefore decided to work on controlling the microstructure of sol-gel-derived BST thin films.

As reported in the article, 300-nm films with layer thicknesses of 60, 20, and $8 \mathrm{~nm}$ were produced by spin coating at $6000 \mathrm{rpm}$ for $40 \mathrm{~s}$ in air from stoichiometric solutions of barium and strontium acetate in acetic acid mixed with titanium butoxide in acetylacetone. The layer thickness was controlled by the concentration of the solution used for spin coating. Each layer was pyrolized at $350^{\circ} \mathrm{C}$ for $5 \mathrm{~min}$ to remove residual organics and crystallized at $750^{\circ} \mathrm{C}$ for $10 \mathrm{~min}$.

"Our results show that the film with a layer thickness of $60 \mathrm{~nm}$ is cubic with equiaxed grains of 30-50 nm size," said Jian-Gong Cheng, who recently received his PhD degree from the Shanghai Institute of Technical Physics. "This is a result of random nucleation in the bulk of each layer during the crystallizing process. But when we reduced the layer thickness to below $20 \mathrm{~nm}$, the nucleation was mostly controlled by the structure of the underlying layer. We could get layerby-layer homoepitaxial growth, which resulted in large columnar grains." While the film with small equiaxed grains showed poor ferroelectric and dielectric properties, a well-defined hysteresis loop and two peaks in the dielectric constanttemperature curve were observed for the film with large columnar grains.

In the August 14 issue of Applied Physics Letters, the group reported that 